\title{
Composite self-similar solutions for relativistic shocks: The transition to cold fluid temperatures
}

\author{
Margaret Pan ${ }^{1}$ and Re'em Sari ${ }^{2}$ \\ ${ }^{1}$ School of Natural Sciences, Institute for Advanced Study, Princeton, New Jersey 08540, USA \\ ${ }^{2}$ California Institute of Technology, MS 130-33, Pasadena, California 91125, USA \\ and Racah Institute of Physics, Hebrew University, Jerusalem 91904, Israel
}

(Received 15 July 2009; accepted 25 August 2009; published online 13 November 2009)

\begin{abstract}
The flow resulting from a strong ultrarelativistic shock moving through a stellar envelope with a polytropelike density profile has been studied analytically and numerically at early times while the fluid temperature is relativistic - that is, just before and after the shock breaks out of the star. Such a flow should expand and accelerate as its internal energy is converted to bulk kinetic energy; at late enough times, the assumption of relativistic temperatures becomes invalid. Here we present a new self-similar solution for the postbreakout flow when the accelerating fluid has bulk kinetic Lorentz factors much larger than unity but is cooling through $p / n$ of order unity to subrelativistic temperatures. This solution gives a relation between a fluid element's terminal Lorentz factor and that element's Lorentz factor just after it is shocked. Our numerical integrations agree well with the solution. While our solution assumes a planar flow, we show that corrections due to spherical geometry are important only for extremely fast ejecta originating in a region very close to the stellar surface. This region grows if the shock becomes relativistic deeper in the star. (C) 2009 American Institute of Physics. [doi:10.1063/1.3249751]
\end{abstract}

\section{INTRODUCTION}

The energy and Lorentz factor that we expect in the ejecta in supernovae and gamma-ray bursts are important because they constrain the amount of energy that can be deposited in the photons we observe from these explosions. Previous work on the ejecta, notably Ref. 1, uses as a starting point the analytic solutions of Johnson and $\mathrm{McKe}^{2}$ for a planar relativistic shock propagating into cold surroundings: By the time the shock reaches the outer envelope of the star, the likely source of the ejecta, it has accelerated to relativistic speeds and its geometry is planar. Several authors described the relativistic shock's propagation and acceleration through this envelope (see, for example, Refs. 3-5), but the work of Johnson and $\mathrm{McKee}^{2}$ and other analytic work on the flow from a relativistic shock that breaks out of a star ${ }^{6,7}$ show that significant acceleration also occurs after the fluid is shocked. As the hot fluid expands adiabatically, its thermal energy is converted to bulk kinetic energy.

Since all the above authors assumed an ultrarelativistic equation of state for the fluid, the fluid never cools and the final Lorentz factor their solutions predict for the fluid is formally infinite. They avoid this difficulty by following fluid elements in the flow only to the point where the fluid temperature becomes nonrelativistic and approximating the final coasting Lorentz factor as the one given by their solutions at that point. They thus find that the final Lorentz factor of a given fluid element scales as $\gamma_{0}^{1+\sqrt{3}}$, where $\gamma_{0}$ is the Lorentz factor acquired by the fluid when it is shocked. This method cannot accurately account for acceleration that occurs around the time when the fluid cools to nonrelativistic temperatures and can only produce approximate relations for the energy and velocity of the ejecta. While Kikuchi and Shigeyama ${ }^{8}$ relaxed the assumption of an ultrarelativistic fluid in their work on this problem, they cannot completely characterize the acceleration while the fluid is cooling either.

We approach this problem by introducing a new kind of self-similar solution for the cooling and expanding fluid. (A general discussion of self-similar solutions may be found in Ref. 9.) In this solution, we require that the fluid move at relativistic speeds but relax the assumption that the fluid be hot. We place the characteristic position at the point where the fluid temperature is transrelativistic. We thus exploit the self-similarity of the transition between hot and cold fluids in the flow rather than the self-similarity in the acceleration of the hot fluid. Indeed, this flow when taken in its entirety is not self-similar: The size scales that characterize the acceleration and the hot/cold transition evolve with time according to different power laws. In other words, the entire flow is a composite of two distinct self-similar solutions. In Sec. II we summarize the solution for the hot fluid, which gives the initial conditions for this new solution. In Sec. III we derive the new solution, and in Sec. IV we describe the behavior of fluid elements in the composite solution. In Sec. V we explain changes in the flow's behavior for very shallow initial density profiles in the stellar envelope. In Sec. VI we discuss the behavior of the flow at late times and relate the elements' final Lorentz factors and the Lorentz factors to which they were initially shocked. In Sec. VII we find regions of the flow where spherical corrections are important, and in Sec. VIII we summarize our findings and discuss them in the context of previous work. We take the speed of light to be $c=1$ throughout our discussion. 


\section{INITIAL CONDITIONS: BEHAVIOR OF THE HOT FLUID}

We are interested in the behavior at late times of a fluid flow which begins as a relativistic shock propagating through the outer layers of a star with a polytropic envelope. As long as the distance between the front of the flow and the original location of the star's surface is small compared to the star's radius, the geometry is planar. So we seek a self-similar solution to the following hydrodynamic equations representing energy, momentum, and mass conservation:

$$
\begin{aligned}
& \frac{\partial}{\partial t}\left[\gamma^{2}\left(e+\beta^{2} p\right)\right]+\frac{\partial}{\partial x}\left[\gamma^{2} \beta(e+p)\right]=0, \\
& \frac{\partial}{\partial t}\left[\gamma^{2} \beta(e+p)\right]+\frac{\partial}{\partial x}\left[\gamma^{2}\left(\beta^{2} e+p\right)\right]=0, \\
& \frac{\partial}{\partial t}(\gamma n)+\frac{\partial}{\partial x}(\gamma \beta n)=0 .
\end{aligned}
$$

As usual, $t$ here is the time, $x$ is the position, $e$ is the energy density, $p$ is the pressure, $n$ is the number density, $\gamma$ is the fluid bulk Lorentz factor, and $\beta$ is the associated fluid bulk velocity. The solution we seek must be connected to the hot flow, whose behavior is well understood: $\mathrm{Sari}^{5}$ and Nakayama and Shigeyama ${ }^{6}$ derived the self-similar solution before the shock breaks out of the star and Pan and Sari ${ }^{7}$ derived the postbreakout solution. Here we simply state these results for reference. We take $x$ to be the position variable relative to the unshocked stellar surface; $x<0$ between the star's center and the location of this unshocked surface. We take $R(t)$ to be the characteristic position in the solution, and we set $R=0$ and $t=0$ at breakout. We take $\Gamma, P$, and $N$ to be the characteristic Lorentz factor, pressure, and number density; $R$ and $\Gamma$ are related in that $\dot{R}=\sqrt{1-1 / \Gamma^{2}} \simeq 1-1 /\left(2 \Gamma^{2}\right)$. We define

$$
\frac{t \dot{\Gamma}}{\Gamma}=-\frac{m}{2}, \quad \frac{t \dot{P}}{P}=-m-k, \quad \frac{t \dot{N}}{N}=-\frac{m}{2}-k .
$$

Here $k$ gives the unshocked density profile in the stellar envelope: Assuming that gravity is constant in the star's outermost layers and unshocked pressure and density are related by a power law, the density is given by a power law $\rho$ $\propto|x|^{-k}$. This implies $-3 \leq k \leq-3 / 2$ for degenerate and convective envelopes and $k=-17 / 13$ for Kramers' opacity envelopes. We consider here the regime $k<-(1+\sqrt{3}) / 3$, which includes all of these profiles; we explain this choice of maximum $k$ in Sec. III. We write the solutions in the form

$$
\begin{aligned}
& \gamma^{2}(x, t)=\frac{1}{2} \Gamma^{2}(t) g(\chi), \\
& p(x, t)=P(t) f(\chi), \\
& n(x, t)=N(t) \frac{h(\chi)}{g^{1 / 2}(\chi)},
\end{aligned}
$$

where the similarity variable ${ }^{10}$ is

$$
\chi=1+2(m+1) \frac{R-x}{R / \Gamma^{2}} .
$$

Note that this expression is equivalent to

$$
\chi=\frac{t-x}{t-R}
$$

taken in the limit where $\Gamma \gg 1$, or where $t \simeq R\{1+1 /[2(\mathrm{~m}$ $\left.+1) \Gamma^{2}\right]$. In other words, the flow's characteristic length scale is $t-R=R /\left[2(m+1) \Gamma^{2}\right]$, and the natural zero point of the position is $x=t$, the location of a photon traveling in the positive $x$-direction and located at the unshocked stellar surface at time 0 .

Mathematically, the pre- and postbreakout solutions differ only in the ranges in $\chi$ which apply. They are

$-\infty<\chi<1, \quad-\infty<g \chi<1$ for $t<0 \quad$ (prebreakout),

$\infty>\chi>0, \quad \infty>g \chi>(g \chi)_{0}$ for $t>0$ (postbreakout),

where

$$
(g \chi)_{0}=4+2 \sqrt{3}-2 k \sqrt{3} .
$$

The ranges in $\chi$ follow from Eq. (9) and the definitions of $R$, $\Gamma, P$, and $N$ before and after breakout. $R$ before breakout corresponds to the location of the shock front, and $\Gamma, P$, and $N$ are associated with fluid just behind the front. Then $R$ $<0$ and the hot fluid to which the prebreakout solution applies is located at $x<R$ so $\chi<1$. The shock accelerates as it approaches the stellar surface and encounters increasingly rarefied fluid as per Eq. (4); arbitrarily close to breakout, $\Gamma$ becomes arbitrarily large. After breakout, there is no shock. In this regime $R>0$ and $R, \Gamma, P$, and $N$ are associated with a fluid element which has expanded by a factor of order unity. Fluid at the front edge of the flow originated just under the star's surface and was shocked to and moves with arbitrarily large Lorentz factors, so the front edge has $\chi=0$ and the rest of the flow has $\chi>0$. The corresponding ranges in $g \chi$ follow from Eqs. (13)-(16) given below.

The solutions are then completely specified by an expression for $m$ in terms of $k$, which gives the time evolution of the flow, and expressions for $g, f$, and $h$ in terms of $\chi$, which give the spatial profiles for the hydrodynamic variables,

$$
\begin{aligned}
& m=(3-2 \sqrt{3}) k, \\
& g=\left|\frac{g \chi-(g \chi)_{0}}{-1+(g \chi)_{0}}\right|^{-(3-2 \sqrt{3}) k},
\end{aligned}
$$




$$
\begin{aligned}
f= & \left|\frac{g \chi-(g \chi)_{0}}{-1+(g \chi)_{0}}\right|^{-(4-2 \sqrt{3}) k}, \\
h= & \left|\frac{-g \chi+(g \chi)_{0}}{1-(g \chi)_{0}}\right|^{-\{[(2 \sqrt{3}-3)(2 k-1) k] /(-1+k \sqrt{3}-\sqrt{3})\}} \\
& \times|g \chi-2|^{k /(-1+k \sqrt{3}-\sqrt{3})} .
\end{aligned}
$$

Thus defined, Eqs. (5)-(7) satisfy the hydrodynamic equations with the equation of state $p=e / 3$. They are an accurate description of the flow with $k<-(1+\sqrt{3}) / 3$ only where the fluid is hot, or where $p / n \gg 1$.

\section{SELF-SIMILAR SOLUTION FOR THE COOLING FLUID}

As the fluid expands and accelerates after breakout, it cools adiabatically from the back of the flow toward the front. At late times, then, the postbreakout solution of Sec. II given in Eqs. (4) and (10)-(16), which we will refer to here as the "hot solution," holds only for a region at the very front of the flow, and this region shrinks with time. The hot solution sets the boundary conditions for the new solution we seek: As we approach the vacuum interface at $\chi=0$, the two solutions must coincide.

In the new solution, which we will refer to as the "cooling solution," we must include cold fluid. We therefore use the equation of state

$$
p=\frac{1}{3}(e-n)
$$

rather than the ultrarelativistic $p=e / 3$. Although this equation of state applies only to fluids with adiabatic index 4/3, our analysis can be easily modified to accommodate an arbitrary equation of state of the form

$$
\frac{p}{n}=F\left(\frac{e}{n}\right)
$$

where $F$ is an invertible function since the hydrodynamic equations can be written in self-similar form with any such equation of state.

We must also specify a characteristic scale and define the characteristic Lorentz factor, pressure, and number density to be consistent with this scale. We seek the profiles of the hydrodynamic variables in the region where the fluid temperature transitions from hot to cold; the natural scale for this transition is the distance $\delta$ between the vacuum interface, where the fluid is hottest, and the point where the fluid temperature becomes nonrelativistic. We set this point to be where $p / n=1$. Then the similarity variable is

$$
\xi=\frac{t-x}{\delta}
$$

and by analogy with the hot solution, we express $\gamma, p$, and $n$ as

$$
\begin{aligned}
& \gamma^{2}(x, t)=\frac{1}{2} \bar{\Gamma}^{2}(t) \bar{g}(\xi), \\
& p(x, t)=\bar{P}(t) \bar{f}(\xi),
\end{aligned}
$$

$$
n(x, t)=\bar{N}(t) \frac{\bar{h}(\xi)}{\bar{g}^{1 / 2}(\xi)} .
$$

We choose $\bar{\Gamma}, \bar{P}$, and $\bar{N}$, the new characteristic values of the Lorentz factor, pressure, and number density, to match the $\gamma$, $p$, and $n$ values, respectively, given by the hot solution where $p / n=1$. With $\chi_{\text {cold }}$ as the value of the old similarity variable $\chi$ corresponding to $p / n=1$ in the hot solution,

$$
\begin{aligned}
& \bar{\Gamma}^{2}=\Gamma^{2} g\left(\chi_{\text {cold }}\right), \\
& \bar{P}=P f\left(\chi_{\text {cold }}\right), \\
& \bar{N}=N \frac{h\left(\chi_{\text {cold }}\right)}{g^{1 / 2}\left(\chi_{\text {cold }}\right)} .
\end{aligned}
$$

This choice of characteristic values dictates

$$
\bar{P}=\bar{N} .
$$

Note that Eqs. (23)-(25) do not fix the values of $\gamma, p$, and $n$ at $p / n=1$ in the cooling solution to those given by the hot solution. Indeed, we know that the true $\gamma, p$, and $n$ of the flow at $p / n=1$ are different from the values given by the hot solution there since the ultrarelativistic equation of state, which the hot solution assumes, is not valid there. Equations (23)-(25) are simply a self-consistent way of choosing a normalization for the characteristic values $\bar{\Gamma}, \bar{P}$, and $\bar{N}$ and tying them to the hot solution. Because we have not fixed the values of $\bar{g}, \bar{f}$, and $\bar{h}$, the values of $\gamma, p$, and $n$ in the cooling solution remain unconstrained.

In the limit of late times, when $\delta \ll R / \Gamma^{2}$ and $g \chi$ $-(g \chi)_{0} \ll(g \chi)_{0}$, Eqs. (23) and (9) give

$$
\begin{aligned}
& (g \chi)_{0} \frac{\Gamma^{2}}{\bar{\Gamma}^{2}} \simeq \chi_{\text {cold }} \simeq \frac{\delta}{t-R} \simeq \delta \frac{2(m+1) \Gamma^{2}}{t}, \\
& \delta=\frac{(g \chi)_{0}}{2(m+1)} \frac{t}{\bar{\Gamma}^{2}}=(2+\sqrt{3}) \frac{t}{\bar{\Gamma}^{2}} .
\end{aligned}
$$

Note also that the characteristic scale $t-R \sim R / \Gamma^{2}$ in the postbreakout solution for the hot fluid is different from the new scale $\delta . R$ is the location of a fluid element that has expanded by a factor of order unity since breakout. Because $R$ evolves according to the finite characteristic Lorentz factor $\Gamma, R$ lags farther and farther behind the front of the flow, where the Lorentz factors are arbitrarily large. In other words, since $\Gamma$ decreases with time as per Eq. (4), $t-R$ increases with time. In the limit of late times, then, $R$ lags far behind the portion of the flow where the fluid remains hot and $t-R$ becomes much larger than the space occupied by the hot fluid-so the scale $t-R$ that characterizes the hot solution becomes irrelevant to the transition between hot and cold fluids that is of interest here.

To get $\bar{\Gamma}$ and $\delta$ as functions of time, we apply Eqs. (14)-(16) at the point $p / n=1$. We use $P / N=\Gamma /(3 \sqrt{2})$, a relation that follows from the shock jump conditions applied in the prebreakout solution, 


$$
\begin{aligned}
1=\frac{p}{n}= & \frac{\Gamma}{3 \sqrt{2}} \frac{\sqrt{g\left(\chi_{\text {cold }}\right)} f\left(\chi_{\text {cold }}\right)}{h\left(\chi_{\text {cold }}\right)} \\
= & \frac{\Gamma}{3 \sqrt{2}}\left[g\left(\chi_{\text {cold }}\right)\right]^{(1 / m)\{(3 m / 2)+k+[m(2 k-1) /(-1-\sqrt{3}+k \sqrt{3})]\}} \\
& \times\left[g\left(\chi_{\text {cold }}\right) \chi_{\text {cold }}-2\right]^{-k /(-1-\sqrt{3}+k \sqrt{3})}, \\
\bar{\Gamma}^{2}= & \Gamma^{2} g\left(\chi_{\text {cold }}\right) \\
= & \Gamma^{2}\left\{\frac{3 \sqrt{2}}{\Gamma}\right. \\
& \left.\times\left[g\left(\chi_{\text {cold }}\right) \chi_{\text {cold }}-2\right]^{k /(-1-\sqrt{3}+k \sqrt{3})}\right\} m /\{(m / 2)-[k /(-1-\sqrt{3}+k \sqrt{3})]\}
\end{aligned} .
$$

This gives

$$
\begin{aligned}
& a=\frac{t \dot{\bar{\Gamma}}}{\bar{\Gamma}}=\frac{k \sqrt{3}}{1+\sqrt{3}+3 k}, \\
& \frac{t \dot{\delta}}{\delta}=\frac{1+\sqrt{3}+(3-2 \sqrt{3}) k}{1+\sqrt{3}+3 k}
\end{aligned}
$$

at late times, when $g\left(\chi_{\text {cold }}\right) \chi_{\text {cold }} \simeq(g \chi)_{0}$. Solving Eq. (29) for $f\left(\chi_{\text {cold }}\right)$ or $h\left(\chi_{\text {cold }}\right) / \sqrt{g\left(\chi_{\text {cold }}\right)}$ similarly gives

$$
b=\frac{t \dot{\bar{P}}}{\bar{P}}=\frac{t \dot{\bar{N}}}{\bar{N}}=-\frac{4 k}{1+\sqrt{3}+3 k} .
$$

Note that $k=-(1+\sqrt{3}) / 3 \simeq-0.91$ makes $a$ and $b$ diverge in Eqs. (33) and (35). This $k$ marks a qualitative change in the behavior of the flow which we discuss further in Sec. V.

Note also that although we defined $\delta, \bar{\Gamma}, \bar{P}$, and $\bar{N}$ with reference to the point $p / n=1$, we could equally well have chosen a reference corresponding to some other $p / n$ of order unity. Choosing another such reference value shifts the values of $\delta, \bar{\Gamma}, \bar{P}$, and $\bar{N}$ by factors of order unity but does not affect Eq. (28) or the time evolution relations in Eqs. (33)-(35). Since the form of the similarity variable $\xi$ is unaffected by a shift in the reference $p / n$, the similarity solution must likewise remain the same as the one we discuss below.

We now proceed to solve the hydrodynamic equations for the relevant range in $k$. We use the equation of state (17) to rewrite Eqs. (1) and (3), and the difference equation obtained by subtracting Eq. (2) from Eq. (1). We take the limit $\gamma \gg 1$. We rewrite the differentiation operators as

$$
\begin{aligned}
& \frac{\partial}{\partial t}=\dot{\bar{\Gamma}} \frac{\partial}{\partial \bar{\Gamma}}+\dot{P} \frac{\partial}{\partial P}+\dot{N} \frac{\partial}{\partial N}+\frac{1}{\delta}(1-\xi \dot{\delta}) \frac{\partial}{\partial \xi}, \\
& \frac{\partial}{\partial x}=-\frac{1}{\delta} \frac{\partial}{\partial \xi},
\end{aligned}
$$

and substitute these and Eqs. (23)-(26), (33), and (35) to get

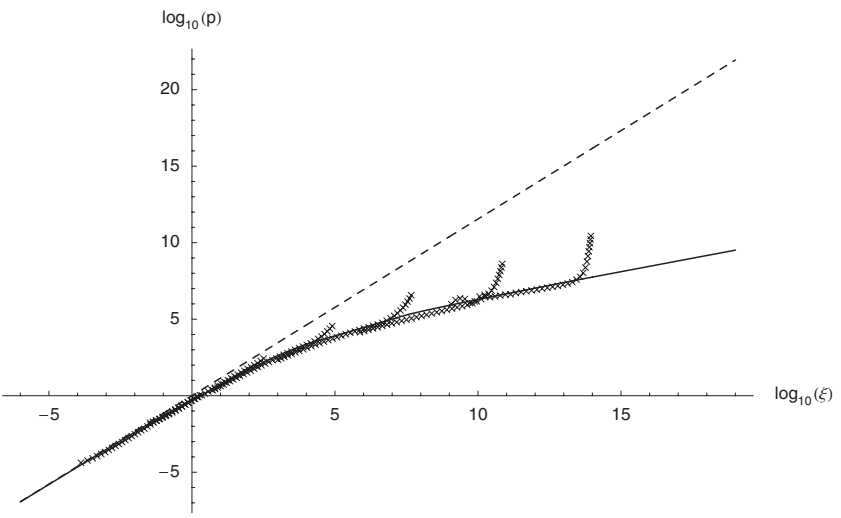

FIG. 1. Profile of the pressure $p$ as a function of the similarity variable $\xi$ for $k=-3$. The dashed line is the hot solution valid for the fluid near the front, at small $\xi$; the solid line is the cooling solution. Numerical simulation data are shown as crosses. In order to cover a substantial range in $\xi$, data from six $p$ vs $\xi$ profiles corresponding to snapshots of the flow at six different times in the same simulation run are shown. The data agree well with the cooling solution. The "tails" at the ends of the numerical simulation profile data are due to edge effects at the ends of the simulation grid that are not self-similar. The overall $y$-axis normalization is arbitrary, but the relative normalizations of the hot solution, the cooling solution, and the numerical simulations are correct. See Sec. IV for further discussion of the relationship between the hot and cooling solutions.

$$
\begin{aligned}
0= & b\left(2 \bar{f}+\frac{\bar{h}}{\bar{g}^{1 / 2}}\right)+\frac{t}{\delta \bar{\Gamma}^{2}}\left[-\bar{g}^{\prime}\left(4 \frac{\bar{f}}{\bar{g}^{2}}+\frac{3}{2} \frac{\bar{h}}{\bar{g}^{5 / 2}}\right)+\frac{4 \bar{f}^{\prime}}{\bar{g}}+\frac{\bar{h}^{\prime}}{\bar{g}^{3 / 2}}\right] \\
& -\xi \frac{t \dot{\delta}}{\delta}\left[-\frac{\bar{g}^{\prime}}{2} \frac{\bar{h}}{\bar{g}^{3 / 2}}+2 \bar{f}^{\prime}+\frac{\bar{h}^{\prime}}{\bar{g}^{1 / 2}}\right], \\
0= & (2 a+b)\left(2 \bar{g} \bar{f}+\frac{\bar{h}^{1 / 2}}{2}\right)+\frac{t}{\delta \bar{\Gamma}^{2}}\left[-\frac{\bar{g}^{\prime}}{4} \frac{\bar{h}}{\bar{g}^{3 / 2}}+\bar{f}^{\prime}+\frac{\bar{h}^{\prime}}{2 \bar{g}^{1 / 2}}\right] \\
& -\xi \frac{t \dot{\delta}}{\delta}\left[\overline{g^{\prime}}\left(2 \bar{f}+\frac{\bar{h}}{4 \bar{g}^{1 / 2}}\right)+2 \bar{f}^{\prime} \bar{g}+\frac{\bar{h}^{\prime}}{2} \bar{g}^{1 / 2}\right], \\
0= & (a+b) \bar{h}+\frac{t}{\delta \bar{\Gamma}^{2}}\left[-\bar{g}^{\prime} \frac{\bar{h}}{\bar{g}^{2}}+\frac{\bar{h}^{\prime}}{\bar{g}}\right]-\xi^{\frac{t}{\delta}} \bar{h}^{\prime} .
\end{aligned}
$$

We substitute Eqs. (28) and (34) into Eqs. (38)-(40) and integrate this ordinary differential equation (ODE) system numerically to produce the solution shown in Figs. 1-3. These figures also compare our solution to a onedimensional numerical simulation of a relativistic planar shock wave which accelerates through and breaks out of a $k=-3$ medium and then expands and cools through the transrelativistic regime. The initial conditions used in the simulation represent the shock as an unresolved contact discontinuity with $\Gamma=5$ at $x=-10^{-5}$ at starting time $t=-1.0$ $\times 10^{-5}$. The simulation grid contains 400 fluid elements spread logarithmically over two orders of magnitude in $x$; our simulation follows these elements until time $t=2.6$ $\times 10^{8}$.

We can check that the behavior of this solution at large $\xi$-where the fluid is very cold and where the hot solution and cooling solution differ most-is physical. Consider a fluid element many distance scales $\delta$ behind the vacuum in- 


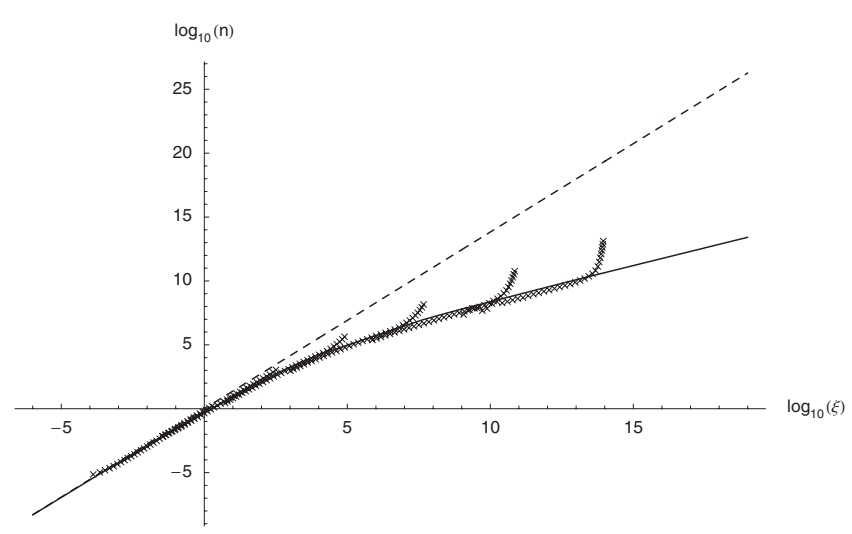

FIG. 2. Same as Fig. 1 for the number density $n$ rather than $p$.

terface at position $t-x \gg \delta$. This fluid element must have become cold at some time $t_{\text {cold }} \ll t$; as a result, it has long since stopped accelerating and has spent most of the time interval $t-t_{\text {cold }}$ coasting at its current Lorentz factor $\gamma$. Then this fluid element has

$$
\begin{aligned}
\xi & =\frac{t-x}{\delta} \simeq \frac{t-\left(t-t_{\mathrm{cold}}\right) \sqrt{1-1 / \gamma^{2}}}{\delta} \simeq \frac{t /\left(\bar{\Gamma}^{2} \bar{g}\right)}{t / \bar{\Gamma}^{2}(2+\sqrt{3})} \\
& =\frac{2-\sqrt{3}}{\bar{g}}
\end{aligned}
$$

so at large $\xi$ we expect

$$
\bar{g} \xi=2-\sqrt{3} \text {. }
$$

We cannot get exact relations for $\bar{f}$ and $\bar{h}$ in the large $\xi$ limit in this way because $p$ and $n$ change significantly while the fluid element finishes its acceleration. However, we can check the scalings of $\bar{f}$ and $\bar{h}$ with $\xi$. Because the fluid elements far behind the front are coasting with Lorentz factors that are virtually constant in time, the volume of each fluid element increases linearly with time. This implies

$$
n \propto t^{-1}, \quad p \propto n^{4 / 3} \propto t^{-4 / 3}
$$

for a single fluid element. From the definitions of $\bar{\Gamma}, \bar{P}$, and $\bar{N}$ in Eqs. (23)-(25), we know $\gamma(\xi=1) / \bar{\Gamma}, p(\xi=1) / \bar{P}$, and

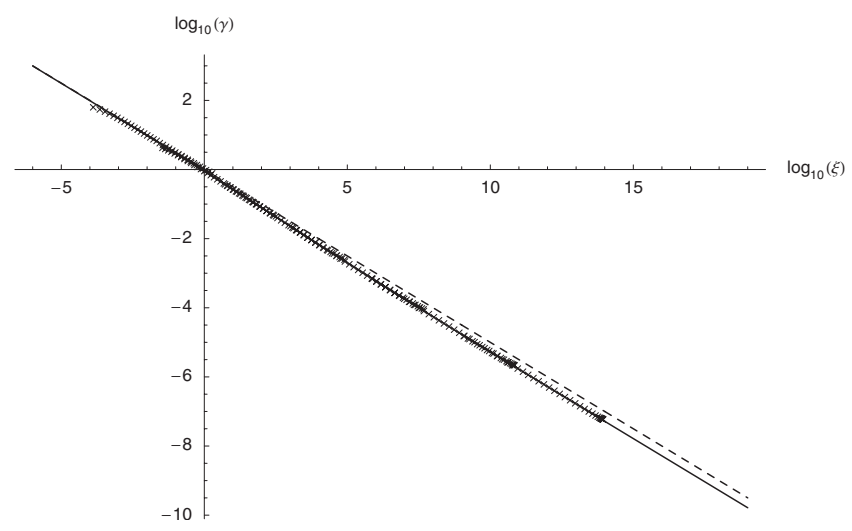

FIG. 3. Same as Fig. 1 for the Lorentz factor $\gamma$ rather than $p$.

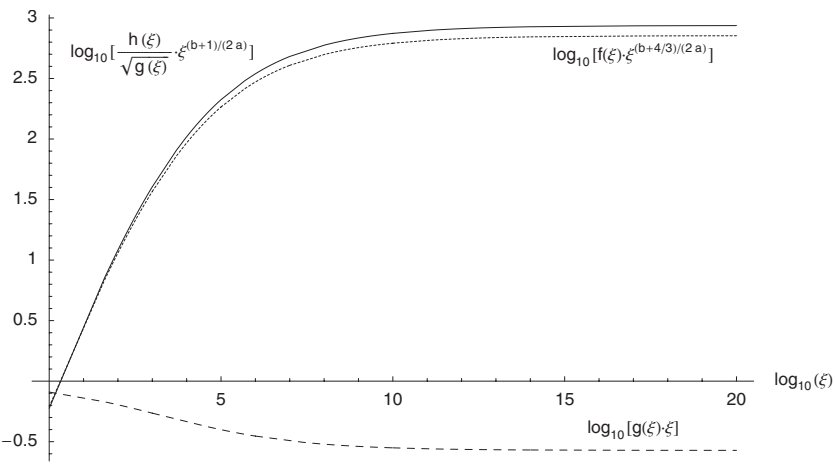

FIG. 4. Verification of the scalings of $\bar{g}, \bar{f}$, and $\bar{h}$ with $\xi$ at large $\xi$, or cold fluid temperatures. The functions plotted (dashed line for $\bar{g}$, dotted line for $\bar{f}$, and solid line for $\bar{h}$ ) were obtained via numerical integration of the ODEs in Eqs. (38)-(40). They show that Eqs. (42), (46), and (47)—relations derived for fluid which has finished accelerating - are valid at large $\xi$. In particular, $\bar{g} \xi$ approaches the expected value $2-\sqrt{3}=10^{-0.572}$. In this calculation we used $k=-3$.

$n(\xi=1) / \bar{N}$, respectively, are constant in time. Then for a single fluid element,

$$
p \sim p\left(t_{\text {cold }}\right)\left(\frac{t}{t_{\text {cold }}}\right)^{-4 / 3} \propto t_{\text {cold }}^{b+4 / 3} .
$$

Since

$$
\bar{g}=\frac{2 \gamma^{2}}{\bar{\Gamma}^{2}(t)} \propto \frac{\bar{\Gamma}^{2}\left(t_{\text {cold }}\right)}{\bar{\Gamma}^{2}(t)} \propto t_{\text {cold }}^{2 a},
$$

we have

$$
\xi \propto t_{\text {cold }}^{-2 a} \propto p^{-2 a /(b+4 / 3)} \rightarrow p \propto \xi^{(b+4 / 3) / 2 a} .
$$

A similar calculation yields

$$
n \propto \xi^{(b+1) / 2 a} .
$$

That the relations in Eqs. (42), (46), and (47) hold at large $\xi$ is shown in Fig. 4.

\section{THE COMPOSITE SOLUTION}

As discussed in Secs. II and III, the hot fluid close to the vacuum interface and the cooling fluid further back in the flow obey two different self-similar solutions in which both the time evolution and the physical interpretation of the characteristic length scale, Lorentz factor, pressure, and density differ. In other words, we describe the entire postbreakout flow-which, when taken as a whole, is not self-similar-by a composite of two self-similar solutions built up around the two different length scales which characterize different portions of the flow. This to our knowledge is the first such "composite solution" found. Chevalier, ${ }^{11}$ for example, also uses two self-similar solutions in a description of a single flow-specifically, the interaction between an expanding shell of fluid and a stationary external medium. However, his solutions have the same characteristic length scale and time evolution; they differ only in the shapes of their profiles and in the disjoint regions of the flow in which they operate. 
They may be considered as a single self-similar solution in which the pressure, velocity, and density profiles are piecewise functions of position.

We can check that the behaviors of fluid elements and sound waves in the hot solution and the cooling solution are consistent. The characteristic position $R$ in the hot solution moves backward relative to the vacuum interface with time because $\Gamma$ decreases with time. By contrast, the characteristic position $t-\delta$ in the cooling solution moves forward relative to $c t$ because fluid elements at the back of the solution cool faster than those at the front, and $t-\delta$ marks the location of a fluid element that has just cooled. We confirm the forward motion by looking at Eq. (34), which indeed gives $t \dot{\delta} / \delta<0$ for the range of $k$ of interest $[k<-(1+\sqrt{3}) / 3]$. We expect fluid elements in the cooling solution to move backward in the solution, or toward larger $\xi$ : Every fluid element must eventually finish accelerating and become cold so the point $t-\delta$ that marks the hot/cold transition must overtake every fluid element. Indeed, the time derivative of $\xi$ following a fluid element,

$$
\frac{D \xi}{D t}=\left(\frac{d}{d t}+\sqrt{1-\frac{1}{\gamma^{2}}} \frac{d}{d x}\right) \xi=\frac{\xi}{t}\left(\frac{2-\sqrt{3}}{\bar{g} \xi}-\frac{t \dot{\delta}}{\delta}\right),
$$

is always positive since $t \dot{\delta} / \delta<0$. Fluid elements in the hot solution move forward with time, toward smaller $g \chi$, since they accelerate while $R$ decelerates. The advective time derivative of $\chi$,

$$
\frac{D \chi}{D t}=\frac{\chi}{t}\left(\frac{2}{g \chi}-1\right)(m+1)
$$

is always negative since $g \chi \geq(g \chi)_{0}>4+2 \sqrt{3}$ everywhere.

If the proper sound speed in the fluid is $\beta_{s}$ $=\sqrt{4 / 3} \bar{f}^{1 / 2} \bar{g}^{1 / 4} \bar{h}^{-1 / 2}$, then the motion of a sound wave in the cooling solution is given by

$$
\begin{aligned}
\frac{d \ln \xi_{ \pm}}{d \ln t} & =\frac{t}{\xi_{ \pm}} \frac{1}{\delta}\left(1-\frac{d x_{ \pm}}{d t}-\xi_{ \pm} \dot{\delta}\right) \\
& =\frac{2-\sqrt{3}}{\bar{g} \xi}\left(\frac{1 \mp \beta_{s}}{1 \pm \beta_{s}}\right)-\frac{t \dot{\delta}}{\delta},
\end{aligned}
$$

where the signs denote forward- and backward-propagating sound waves. Again, $t \dot{\delta} / \delta<0$, so $d \xi_{ \pm} / d t>0$ everywhere and all sound waves move backward in the cooling solution. In other words, all fluid elements are disconnected from the vacuum interface. In the sense that the front is disconnected from the fluid far back in the flow, the cooling solution is similar to type II solutions. However, type II solutions are characterized by their containing a sonic point which is used to find the solution; the sonic point both makes this disconnect explicit and clearly separates connected regions at the front and back of the flow. The cooling solution contains no sonic point.

There is a caveat in this composite view of the flow for the density profiles $k<-(1+\sqrt{3}) / 3$ discussed in Secs. II and III. At early times when $\Gamma \gg 1$, all the fluid elements which have accelerated by at least a factor of order unity are those with $p / n \gtrsim \Gamma$; slower and cooler fluid elements still have

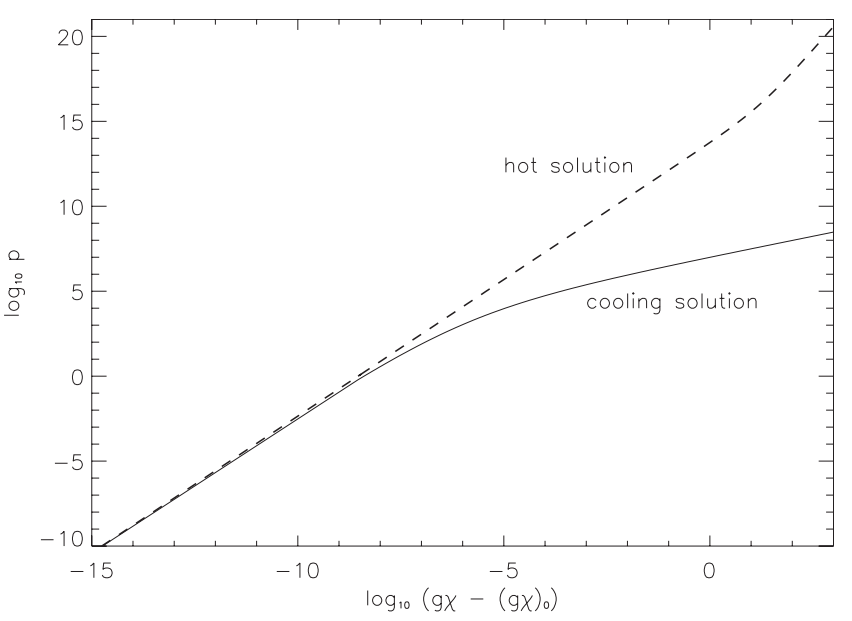

FIG. 5. Pressure profiles for initial density profile $k=-3$ at late times when the cooling solution applies. The dashed curve is the hot solution and the solid curve is the cooling solution. The front of the flow, where $g \chi \simeq(g \chi)_{0}$, is toward the left. As in Fig. 1, the overall $y$-axis normalization is arbitrary but the relative normalizations of the hot and cooling solutions are correct. The "kinks" in the hot and cooling solution curves occur at their respective characteristic scales $t-R$ and $\delta$. Fluid far back in the flow, where $\xi \gg 1$ or, equivalently, $g \chi-(g \chi)_{0} \gg(g \chi)_{\text {cold }}-(g \chi)_{0}$, follows the cooling solution. This cold fluid falls behind the position predicted for it in the hot solution because it is no longer accelerating. As a result, the hot solution gives artificially high pressures for fluid at the back of the flow. The hot solution applies only at the front of the flow in a region small compared to both characteristic scales. As this region corresponds to the limit in which we set the cooling solution to match the hot solution, the entire flow may be described with the cooling solution alone at these times when $\Gamma<1$.

very nearly the same temperatures and speeds as they had just after being shocked. So the only fluid elements with $p / n$ of order unity or smaller are those which were never shocked to relativistic temperatures, and the part of the flow moving relativistically can be described with the hot solution alone. At late times when the cold solution is relevant, the hot solution applies only in a region at the front of the flow whose size is much smaller than the characteristic scale $R / \Gamma^{2}$. In this small region, the profiles of the hydrodynamic variables in hot solution appear very nearly scale free, and the cooling solution approaches the resulting power-law profiles toward the front of the flow, in the limit of small $\xi$. Then the hot solution is unnecessary to the description of the flow at these late times so we can think of the flow as following the hot solution at early times and transitioning to the cooling solution when $\Gamma=1$. Figure 5 is a schematic of the composite flow at these late times. However, a composite of the hot and cooling solutions is essential in describing the flow for density profiles $-(1+\sqrt{3}) / 3 \leq k<0$, which we discuss in Sec. V.

\section{SOLUTIONS WHEN $-(1+\sqrt{3}) / 3 \leq k<0$}

We restricted our discussion of the hot and cooling solutions in Secs. II-IV to initial density profiles $k<-(1$ $+\sqrt{3}) / 3$. That the initial density decrease toward the star's surface requires $k<0$, but so far we have neglected the interval $-(1+\sqrt{3}) / 3 \leq k<0$. To describe the flow for these $k$, we return to the singularity at $k=-(1+\sqrt{3}) / 3$ noted in Sec. III and examine the behavior of $(g \chi)_{\text {cold }}=g\left(\chi_{\text {cold }}\right) \chi_{\text {cold }}$.

Equation (29) implies 


$$
\frac{d \ln (g \chi)_{\text {cold }}}{d \ln t}=-\frac{(g \chi)_{\text {cold }}-(g \chi)_{0}}{(g \chi)_{\text {cold }}} \frac{(g \chi)_{\text {cold }}-2}{(g \chi)_{\text {cold }}-2(3+4 / \sqrt{3})} .
$$

For $-(1+\sqrt{3}) / 3<k<0$, integration of Eq. (51) shows that $(g \chi)_{\text {cold }}$ is a double-valued function of time until the entire flow cools. As $t \rightarrow 0$ from above, $(g \chi)_{\text {cold }} \rightarrow\left\{(g \chi)_{0}, \infty\right\}$; for positive times, the smaller value of $(g \chi)_{\text {cold }}$ increases and the larger value decreases with time until $(g \chi)_{\text {cold }}$ becomes single valued at $(g \chi)_{\text {cold }}=2(3+4 / \sqrt{3})$, which occurs at a finite time. In other words, fluid cools quickly both at the front, where the expansion time scale is shortest, and at the very back, where the shocked fluid is coldest.

Since $(g \chi)_{\text {cold }}$ becomes single valued at a $(g \chi)_{\text {cold }}$ of order unity, the value of $g$ corresponding to the larger value of $(g \chi)_{\text {cold }}$ is always of order unity or smaller. However $g=1$ for a fluid element which has accelerated by a factor of order unity, so this larger $(g \chi)_{\text {cold }}$ tracks fluid elements which cool before or just as they manage to accelerate by a factor of order unity. These fluid elements cannot have been shocked to relativistic temperatures, so we focus on the evolution of the smaller value of $(g \chi)_{\text {cold }}$ as it moves through the front of the solution. In contrast to the $k<-(1+\sqrt{3}) / 3$ case, the fluid near the front cools from the vacuum interface backward, from the fastest-moving toward slower-moving fluid, and the fluid farthest forward is coldest: $\bar{\Gamma}$ decreases with time and $\bar{P}, \bar{N}$, and $\delta$ increase with time. Fluid elements just behind the front-specifically, those with $g \chi-(g \chi)_{0} \ll 2+2 / \sqrt{3}$ $+2 k \sqrt{3}$-always have $(g \chi)_{\text {cold }} \simeq(g \chi)_{0}$, so they obey Eqs. (33)-(35). Then the discussion of the cooling solution in Sec. III applies in the limit of small positive times, when the front of the flow is cooling, except in that the initial conditions used to integrate Eqs. (38)-(40) are set by matching to the hot solution far back in the cooling flow, at $\xi \gg 1$, rather than at the vacuum interface. Between breakout and the time when all the fluid cools, the flow contains both hot and cold fluids moving at relativistic bulk speeds; it therefore follows a true composite of the hot and cooling solutions. Figure 6 shows a schematic of this composite solution.

As in the $k<-(1+\sqrt{3}) / 3$ case, fluid elements move forward in the hot solution with time according to Eq. (49). No qualitative change in the time evolution of the scale $R-$ or, therefore, in the behavior of fluid in the hot solution-occurs as $k$ increases through $-(1+\sqrt{3}) / 3$. In contrast to the $k<-(1+\sqrt{3}) / 3$ case, fluid elements move forward in the cooling solution as well. Equation (41), which still holds in the limit $t-x \ll \delta$, implies $\bar{g} \xi \geq 2-\sqrt{3}$, while Eq. (34) implies $t \dot{\delta} / \delta>1$. Then from Eq. (48) we have $D \xi / D t<0$.

For $k=-(1+\sqrt{3}) / 3, \quad(g \chi)_{0}=3(2+4 / \sqrt{3})$, so $(g \chi)_{\text {cold }}$ is single valued and $(g \chi)_{\text {cold }}-2 \propto t^{-1}$. Then there is a finite time at which $(g \chi)_{\text {cold }}$ reaches $(g \chi)_{0}, \delta$ shrinks to 0 , and the entire flow cools. Also, at any $t>0$, Eqs. (15) and (16) imply that the temperature at the front of the flow, where $g \chi-(g \chi)_{0}$ $\ll(g \chi)_{0}$, is roughly independent of position, and the temperature at the vacuum interface is finite; all the fluid at the front cools at the same rate. Although Eq. (34) does not work for this $k$, we know $\delta$ decreases monotonically to 0 with time so

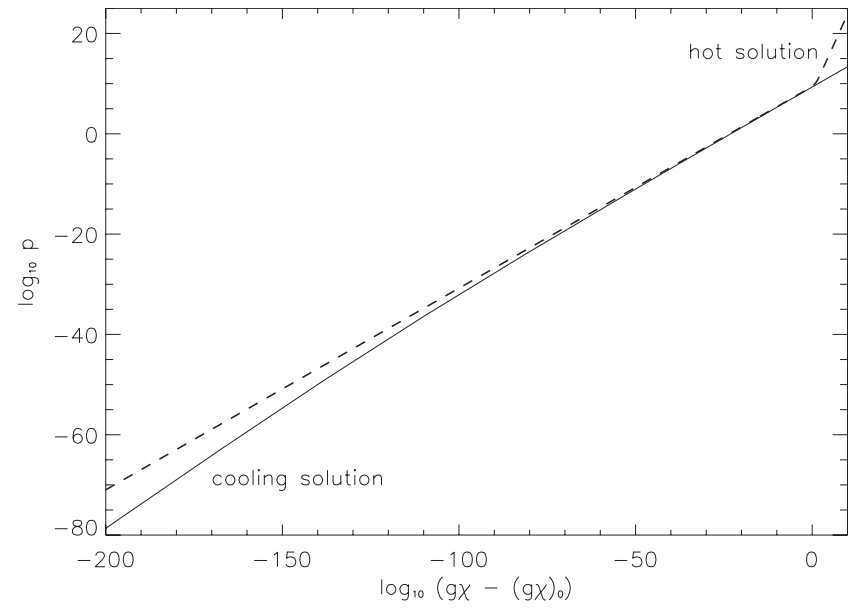

FIG. 6. Same as Fig. 5, but for initial density profile $k=-3 / 4$ at early times, before $(g \chi)_{\text {cold }}$ becomes single valued. Here the cooling solution applies at the front of the flow; the hot solution applies further back in the flow. Because the cold fluid has stopped accelerating, the hot solution gives artificially high pressures for fluid toward the front of the flow where $\xi \ll 1$ or $g \chi-(g \chi)_{0} \ll(g \chi)_{\text {cold }}-(g \chi)_{0}$. However, the cooling solution misses the transition between regions of hot fluid which have and have not accelerated significantly since being shocked. This transition occurs at the characteristic position in the hot solution (Ref. 7) as specified in Sec. II so it is located at $\chi \sim 1$, or $g \chi-(g \chi)_{0}$ of order unity, and corresponds to the "knee" in the hot solution. An accurate description of the entire flow therefore requires both the hot and cooling solutions.

Eq. (48) gives $D \xi / D t>0$. Fluid elements move backward in the cooling solution until $(g \chi)_{\text {cold }}=(g \chi)_{0}$ and $\delta=0$.

\section{BEHAVIOR OF FLUID ELEMENTS AT LATE TIMES}

Earlier analytic work has established that the final Lorentz factor $\gamma_{\text {final }}$ of a given fluid element should scale according to

$$
\gamma_{\text {final }}=K \gamma_{\text {shocked }}^{1+\sqrt{3}},
$$

where $\gamma_{\text {shocked }}$ is the fluid element's Lorentz factor immediately after it is shocked in the prebreakout flow and the coefficient $K$ is independent of $\gamma_{\text {shocked. }}{ }^{2,7}$ Tan et al. ${ }^{1}$ found numerically that $K \simeq 2.6$ for $k=-3$. They note, and we confirm from our own experience, that it is difficult to continue numerical simulations until the very end of the fluid acceleration since the conversion of thermal to bulk kinetic energy is quite slow: Fig. 4 indicates significant acceleration until $\xi \sim 10^{10}$. To estimate the coefficient, Tan et al. ${ }^{1}$ applied correction factors to their simulation results of up to $\sim 50 \%$ for fluid elements with final Lorentz factors of order $\sim 10^{3}$.

We can find $\gamma_{\text {final }}$ for a given fluid element directly from our pre- and postbreakout solutions. To track the acceleration of the fluid element while it is hot, we take the advective time derivative of $\gamma$ in the pre- and postbreakout solutions for the hot fluid,

$$
\begin{aligned}
& \frac{D \gamma}{D t}=\frac{\gamma}{t} \frac{(\sqrt{3}-3) k}{g \chi-4-2 \sqrt{3}}, \\
& \frac{D g \chi}{D t}=\frac{1}{t} \frac{(2-g \chi)\left[g \chi-(g \chi)_{0}\right]}{g \chi-4-2 \sqrt{3}},
\end{aligned}
$$




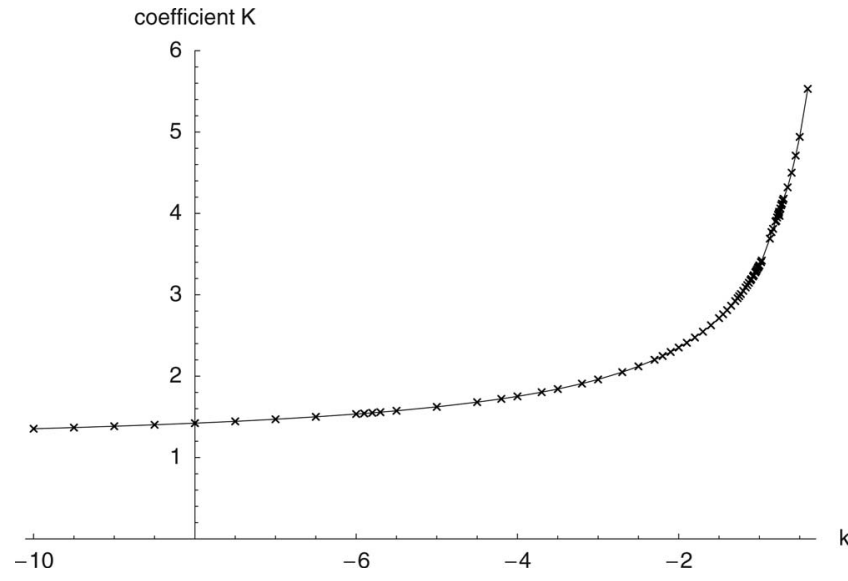

FIG. 7. Coefficient $K$ for the final Lorentz factor as defined in Eq. (52) plotted as a function of density profile $k$. The crosses were computed from the cooling solution; the line connecting them is included to guide the eye.

$$
\frac{D \gamma}{D g \chi}=\gamma \frac{(\sqrt{3}-3) k}{(2-g \chi)\left[g \chi-(g \chi)_{0}\right]} .
$$

We now integrate Eq. (55) with the proper limits. Before breakout, the fluid element's $g \chi$ goes from $g \chi=1$ when it is shocked to $g \chi \rightarrow-\infty$ at breakout. After breakout, the fluid element's $g \chi$ goes from $g \chi \rightarrow \infty$ to some $g \chi \simeq(g \chi)_{\text {cold. }}$. So when the fluid becomes cold, we have

$$
\begin{aligned}
\gamma= & \gamma_{\text {shocked }}\left[(g \chi)_{0}-1\right]^{[(\sqrt{3}-3) k]]\left[(g \chi)_{0}-2\right]} \\
& \times\left[\frac{g \chi-2}{g \chi-(g \chi)_{0}}\right]^{[(\sqrt{3}-3) k] /\left[(g \chi)_{0}-2\right]} .
\end{aligned}
$$

To find the correct $g \chi$ at which to evaluate the above, we set $C=\gamma f / h$ to be the temperature (up to a factor of 3 ) at the $g \chi$ of interest and use Eqs. (15) and (16) to express ( $g X$ $-2) /\left[g \chi-(g \chi)_{0}\right]$ in terms of $C$. This gives

$$
\gamma=C^{-\sqrt{3}} \gamma_{\text {shocked }}^{\sqrt{3}+1}
$$

To this we add the extra factor given by the cooling solution to get the coefficients $K$ shown in Fig. 7. In particular,

$$
\begin{aligned}
& \gamma_{\text {final }}=1.96 \gamma_{\text {shocked }}^{\sqrt{3}+1}, \quad k=-3, \\
& \gamma_{\text {final }}=2.71 \gamma_{\text {shocked }}^{\sqrt{3}+1}, \quad k=-3 / 2 .
\end{aligned}
$$

This result is close to the results of Tan et al., ${ }^{1}$ who found a coefficient of $\sim 2.6$ when $k=-3$. Note that $p / n=1$ corresponds to $C^{-\sqrt{3}}=0.149: \gamma$ grows by a factor of $\sim 15$ after the fluid element becomes nominally cold.

The growth of $\gamma$ as a function of the temperature for a single fluid element is shown in Fig. 8, which also shows good agreement between the cooling solution and direct numerical simulations of the hydrodynamic equations. Because the Lorentz factors near the front of the flow, in particular, become very large at late times, it is difficult to produce numerical simulations that remain accurate as the fluid cools all the way to $p / n \ll 1$. As a result, the numerical simulation shown in Fig. 8 cuts off while the fluid Lorentz factor is $9 \%$ smaller than the final Lorentz factor predicted by the cooling solution.

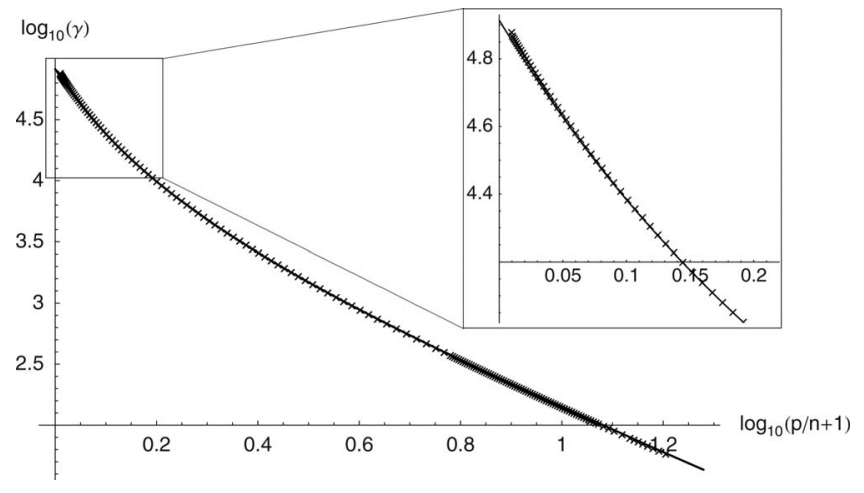

FIG. 8. Lorentz factor $\gamma$ of a single fluid element as a function of the temperature $p / n$ of that fluid element. Lower temperatures and later times are toward the left. The solid line is the self-similar solution; the points are the results of numerical simulations. Both calculations were done for $k=-3$. As the fluid element becomes cold, the evolution of its $\gamma$ with $p / n$ deviates from the power law seen at high temperatures.

\section{EFFECTS OF SPHERICAL GEOMETRY}

We can estimate the ranges of initial positions and Lorentz factors for which corrections to our planar solutions due to the star's spherical geometry are important. Spherical geometry significantly affects a given fluid element's acceleration if the distance between the fluid element and the star's center doubles before the fluid element finishes accelerating. Once the fluid element has traveled a distance comparable to $R_{*}$, the star's radius, it has expanded significantly in directions perpendicular to its motion; planar solutions cannot account for this transverse expansion. For our estimate we therefore check which fluid elements have cooled to $p / n$ $\sim 1$ by time $t \sim R_{*}$.

Consider a fluid element whose position before being shocked is $x_{\text {init }}<0$. According to the relativistic shock jump conditions, immediately after this fluid element is shocked it has

$$
\left(\frac{p}{n}\right)_{\text {init }} \sim \gamma_{\text {init }} \sim \Gamma \sim\left(\frac{x_{\text {init }}}{x_{\text {rel }}}\right)^{-m / 2},
$$

where $\Gamma$ is the shock Lorentz factor in the prebreakout solution and $x_{\text {rel }}$ is the position of the shock when it first becomes relativistic, or when $\Gamma \simeq \sqrt{2}$. Right after breakout our fluid element follows the hot solution; when it reaches $\chi=1$, it has accelerated and expanded by a factor of order unity since being shocked so it still has $p / n \sim\left(x_{\text {init }} / x_{\text {rel }}\right)^{-m / 2}$. Also, at this point $\Gamma \sim \gamma_{\text {init }}$ so the time is $\sim\left|x_{\text {init }}\right|$. We track the temperature of the fluid element over time until it reaches $p / n=1$ by taking the advective time derivative of $p / n$,

$$
\frac{D \ln (p / n)}{D \ln t}=\frac{k(\sqrt{3}-1)}{g \chi-4-2 \sqrt{3}} \simeq \frac{\sqrt{3}-3}{6},
$$

where the final equality holds while the fluid element is at $g \chi-(g \chi)_{0} \ll(g \chi)_{0}$ - that is, near the front of the flow, where most of the acceleration takes place. We then have 


$$
\left(\frac{p}{n}\right) \sim\left(\frac{p}{n}\right)_{\text {init }}\left(\frac{t}{\mid x_{\text {init }}}\right)^{(\sqrt{3}-3) / 6} .
$$

We now impose the condition $p / n \sim 1$ at or before $t \sim R_{*}$ to get the scaling

$$
\frac{\left|x_{\text {rel }}\right|}{R_{*}}<\left(\frac{\left|x_{\text {init }}\right|}{R_{*}}\right)^{1+[(1+\sqrt{3}) / 3 k]} .
$$

Sphericity corrections are unimportant for fluid elements which satisfy this condition. For $0>k \geq-(1+\sqrt{3}) / 3$, the exponent on the right hand side is not positive so spherical corrections are unimportant as long as $\left|x_{\text {rel }}\right|<R_{*}$. On the other hand, for $k<-(1+\sqrt{3}) / 3$ spherical corrections are important for a layer of fluid initially adjacent to the star's surface: In our self-similar solutions for these $k$ values, fluid elements that start arbitrarily close to the star's surface will take arbitrarily long to cool.

The equivalent condition on $\gamma_{\text {init, }}$, the Lorentz factor of a given fluid element just after being shocked, is

$$
\frac{\left|x_{\text {rel }}\right|}{R_{*}}<\left(\gamma_{\text {init }}\right)^{-(1+\sqrt{3}+3 k) /[(3+\sqrt{3}) k]},
$$

the one for $\gamma_{\text {final }}$, the fluid element's Lorentz factor after it finishes accelerating, is

$$
\frac{\left|x_{\text {rel }}\right|}{R_{*}}<\left(\gamma_{\text {final }}\right)^{-(1+\sqrt{3}+3 k) / \sqrt{3} k} .
$$

\section{SUMMARY AND DISCUSSION}

We have derived a new self-similar solution, the cooling solution, for the flow that results when a relativistic shock breaks out of a polytropic envelope. The cooling solution is based on our identification of the characteristic position with the point where the fluid cools to nonrelativistic temperatures. The cooling solution shows that the transition between hot and cold fluids in the flow is self-similar even though this transition is not included-indeed, is not self-similar-in the old postbreakout solution for the hot fluid alone. We present a description of a non-self-similar flow using a composite of two distinct self-similar solutions in which the time evolution and physical interpretation of the characteristic scales differ. As the envelope's original density profile $k$ increases past $k=-(1+\sqrt{3}) / 3$, the flow dictated by the composite solution changes qualitatively from one where the slowest fluid at the back of the flow cools most quickly to one where the fastest fluid at the front of the flow cools most quickly.

The cooling solution allows accurate calculation of the final Lorentz factors of the shocked fluid elements. Given a stellar model for a core-collapse supernova progenitor and an input explosion energy, we can extract the initial density profile of the progenitor envelope and the initial shock velocity and use the cooling solution to find the Lorentz factor and kinetic energy profiles in the relativistic ejecta after the ejecta finish accelerating. This provides an accurate value for the energy available to produce observable light curves via interaction between the ejecta from the model explosion and the progenitor's surroundings.
Kikuchi and Shigeyama ${ }^{8}$ also investigate cooling in the flow produced after a relativistic shock breakout. They focus on a $k=-3$ density profile and use as starting point the work of Nakayama and Shigeyama, ${ }^{6}$ who found a numerical selfsimilar solution for the hot planar flow in a Lagrangian framework. While Kikuchi and Shigeyama ${ }^{8}$ also describe the cooling flow with a system of ODEs, they conclude that no self-similar solution exists for the cooling flow: Instead of adopting a new characteristic scale in writing the ODEs for the cooling flow, they retain the characteristic scale relevant to the hot solution. Because they do not recognize the selfsimilarity of the flow and because they do not integrate until the fluid has cooled enough, they find that initially hot fluid does not stop accelerating. As a result they cannot express the final Lorentz factors of given fluid elements in terms of the initial ones.

In addition, Kikuchi and Shigeyama ${ }^{8}$ focus on corrections to their planar flow due to spherical geometry. They perturb the hydrodynamical equations to lowest order in a new variable equal to the position coordinate scaled to the stellar radius. Their non-self-similar solutions to the perturbed equations agree well with their numerical simulations only at very early times, before the fluid has expanded by a factor of $\sim 2$. They look at sphericity effects in the cooling flow using numerical simulations and find that for fluid whose initial fractional distances from the star's surface to its center lie between about $4 \times 10^{-4}$ and $3 \times 10^{-3}$, sphericity effects change the final velocities by factors of order unity in simulations with $k=-3, \Gamma=10^{5}$ and $10^{6}$ at $t=1$, and $R_{*}=3.3$ in units where $c=1$. Although such fast shocks are unrealistic since they would have $\Gamma \sim 10^{4}$ or $10^{5}$ at the star's center, our scaling in Sec. VII also indicates that sphericity corrections would be important for these parameters.

Tan et al. ${ }^{1}$ also consider sphericity corrections for the density profile $k=-3$, but they treat the opposite limit of mildly relativistic shocks with $\Gamma-1$ ranging from about 7 $\times 10^{-3}$ to 0.28 when the shock is halfway from the star's center to its surface. Only their two most energetic numerical calculations of sphericity effects attain shock Lorentz factors $\Gamma>\sqrt{2}$ so nonrelativistic estimates of sphericity corrections are relevant for most of the regime they consider. However, those two most energetic shock calculations show that for the relativistic fluid elements shocked to initial Lorentz factors between about $\sqrt{2}$ and 4 , sphericity decreases the final velocities by $20 \%-30 \%$. Our scaling in Sec. VII indicates that for those two scenarios, sphericity corrections should be important for fluid elements shocked to Lorentz factors larger than about 1.7 and 2.4, respectively; this is roughly consistent with the findings of Tan et al. ${ }^{1}$

\section{ACKNOWLEDGMENTS}

This research was partially funded by an IRG grant, a NASA ATP grant, and NSF Grant No. PHY-0503584. M.P. thanks Frank and Peggy Taplin and the Association of Members of the Institute for Advanced Study for support. R.S. is a Packard Fellow and an Alfred P. Sloan Research Fellow. 
${ }^{1}$ J. C. Tan, C. D. Matzner, and C. F. McKee, "Trans-relativistic blast waves in supernovae as gamma-ray burst progenitors," Astrophys. J. 551, 946 (2001).

${ }^{2}$ M. H. Johnson and C. F. McKee, "Relativistic hydrodynamics in one dimension,” Phys. Rev. D 3, 858 (1971).

${ }^{3}$ P. Best and R. Sari, "Second-type self-similar solutions to the ultrarelativistic strong explosion problem," Phys. Fluids 12, 3029 (2000).

${ }^{4}$ R. Perna and M. Vietri, "A self-similar solution for the propagation of a relativistic shock in an exponential atmosphere," Astrophys. J. Lett. 569, L47 (2002).

${ }^{5}$ R. Sari, "First and second type self-similar solutions for implosions and explosions containing ultrarelativistic shocks," Phys. Fluids 18, 027106 (2006).
${ }^{6}$ K. Nakayama and T. Shigeyama, "Self-similar evolution of relativistic shock waves emerging from plane-parallel atmospheres," Astrophys. J. 627, 310 (2005)

${ }^{7}$ M. Pan and R. Sari, "Self-similar solutions for shocks breaking out of stars with polytropic envelopes," Astrophys. J. 643, 416 (2006).

${ }^{8}$ R. Kikuchi and T. Shigeyama, "Relativistic flows after shock emergence," Astrophys. J. 657, 860 (2007).

${ }^{9}$ Ya. B. Zel'dovich and Yu. P. Raizer, Physics of Shock Waves and HighTemperature Phenomena (Academic, New York, 1967).

${ }^{10}$ R. D. Blandford and C. F. McKee, "Fluid dynamics of relativistic blast waves," Phys. Fluids 19, 1130 (1976).

${ }^{11}$ R. A. Chevalier, "Self-similar solutions for the interaction of stellar ejecta with an external medium," Astrophys. J. 258, 790 (1982). 